\title{
Tendência temporal do nascimento pré-termo e de seus determinantes em uma década
}

\author{
Temporal trends of preterm birth \\ and its determinants over a decade
}

Bruna Balbi ${ }^{1}$

Maria Antonieta de Barros Leite Carvalhaes ${ }^{1}$

Cristina Maria Garcia de Lima Parada ${ }^{1}$

${ }^{1}$ Departamento de Enfermagem, Faculdade de Medicina de Botucatu, Universidade Estadual Paulista Júlio de Mesquita Filho. Campus Universitário de Rubião Júnior s/n, Rubião Júnior. 18618970 Botucatu SP Brasil. cparada@fmb.unesp.br

\begin{abstract}
This study sought to analyze the frequency of preterm births and identify the respective risk factors and their evolution over a decade (2001-2005-2010) in a city in São Paulo state. It is a time-series study using data from the Live Birth Information System. Univariate and multiple logistic regression were used to identify factors associated with preterm births, and linear regression was used to evaluate the time-series tendency of such factors in the period. To avoid underestimation, a correction factor was applied to the preterm frequencies obtained. A discrete increase in preterm birth was observed: $12.5 \%, 12 \%$ and 13.2\%. After adjusting for confounding factors, maternal age equal to or higher than 35 years and cesarean sections were associated with higher chances for preterm births and increased over the decade. The number of births in high-risk hospitals and of women with fewer than seven prenatal consultations decreased during the period, while multiple pregnancies remained stable. Compared to vaginal births, cesarean sections doubled the chance of preterm birth. Among the five factors associated with preterm birth, three (cesarean sections, births in high-risk hospitals and fewer than seven prenatal consultations) can be modified by actions taken by health care services.
\end{abstract}

Key words Premature, Pregnancy, Epidemiology, Information systems
Resumo Objetivou-se analisar a frequência de nascimentos pré-termo, identificar fatores de risco e a evolução destes em uma década (2001-20052010) em município paulista. Estudo de série temporal realizado com dados do Sistema de Informações de Nascidos Vivos. Utilizou-se a regressão logística univariada e múltipla para identificar fatores associados ao nascimento pré-termo e a regressão linear para avaliar a tendência temporal destes no período. Para evitar subestimação, às frequências de nascimento pré-termo obtidas aplicou-se fator de correção. Houve discreto aumento da taxa de nascimento pré-termo: 12,5\%, 12,0\% e 13,2\%. Após ajuste para confundidores, associaram-se com maior chance desse desfecho e aumentaram na década: idade materna igual ou superior a 35 anos e parto cesárea; diminuíram no período: nascimento em hospital de alto risco e menos de sete consultas pré-natais, permanecendo estável a gemelaridade. Comparando ao parto vaginal, nascer de cesariana dobrou a chance de nascimento pré-termo. Dentre os cinco fatores associados à prematuridade, três (parto cesárea, nascimento em hospital de alto risco e menos de sete consultas de pré-natais) são modificáveis por ações no âmbito dos serviços de saúde.

Palavras-chave Prematuro, Gravidez, Epidemiologia, Sistemas de informação 


\section{Introdução}

O nascimento pré-termo é importante problema de saúde pública. Dentre as graves consequências deste evento estão a maior chance de morte no período neonatal ${ }^{1}$ e de desenvolvimento de intercorrências graves, como a enterocolite necrotizante $^{2}$, a hemorragia intraventricular ${ }^{3}$, problemas respiratórios $^{4}$ e de desenvolvimento ${ }^{5}$ e hipertensão pulmonar persistente ${ }^{6}$, entre outras, podendo repercutir inclusive na vida adulta.

A etiologia do nascimento pré-termo é complexa e multifatorial ${ }^{1,7}$, sofrendo interferência de fatores sociais, como baixa renda e baixa escolaridade materna ${ }^{8}$; psicológicos, como depressão e ansiedade ${ }^{9}$; comportamentais, como o tabagis$\mathrm{mo}^{10}$; socioeconômico e culturais, incluindo idade materna nos extremos do período e relacionados à assistência pré-natal ${ }^{8} \mathrm{e}$ biológicos, como gemelaridade e malformação ${ }^{11}$.

Em todo mundo, estima-se que ocorram a cada ano 13 milhões de nascimentos pré-termos, porém com importantes diferenças regionais, visto que aproximadamente 11 milhões de casos (85\%) concentram-se na África e Ásia. No total, $9,6 \%$ dos partos são pré-termos, sendo a menor taxa encontrada na Europa $(6,2 \%)$ e a maior na África (11,9\%), estando as taxas em ascensão nas duas últimas décadas em quase todos os países? Recente revisão sistemática apontou que em 2013 ocorreram 6,3 milhões de óbitos de crianças menores de cinco anos, $44 \%$ destes no período neonatal e tendo como principal causa de morte as complicações do parto pré-termo, que pela primeira vez superou a pneumonia ${ }^{12}$.

A situação do nascimento pré-termo no Brasil não deixa o país em situação confortável: em relatório da Organização Mundial de Saúde nos cita entre as 10 nações com maior número de nascimentos pré-termos, com taxas próximas às dos Estados Unidos da América, Índia e Nigéria, demonstrando tratar-se de um problema global. Este relatório indica, ainda, que 9,2\% de todos os partos ocorridos no Brasil em 2010 foram prematuros $^{11}$, valor inferior à prevalência de 11,3\% obtida em estudo nacional de base hospitalar de 2013, sugerindo aumento da taxa de nascimento pré-termo no país em anos recentes ${ }^{13}$.

Conhecer e compreender o complexo processo do nascimento e os fatores que nele interferem, incluindo aqueles que aumentam o risco de parto pré-termo, é fundamental para a oferta de assistência de qualidade e efetiva ao binômio mãefilho. Em particular, monitorar os nascimentos prematuros, identificar seus determinantes e acompanhar a evolução de ocorrência em contextos específicos são medidas importantes para a definição de políticas e intervenções preventivas ou relacionadas ao cuidado à saúde desse grupo.

As disparidades regionais e sociais identificadas na ocorrência e a tendência temporal dos nascimentos pré-termo no Brasil ${ }^{8}$ indicam a pertinência de análises adicionais, desagregadas até o nível municipal. Assim, o presente estudo teve por objetivo analisar a tendência temporal de nascimentos pré-termo, identificar fatores de risco para a prematuridade e avaliar sua frequência ao longo de uma década em município de médio porte do interior paulista.

\section{Métodos}

Realizou-se estudo de série temporal com residentes em Botucatu/SP, município com 131.723 habitantes ${ }^{14}$, localizado na região centro-sul do Estado de São Paulo. A fonte dos dados foi o Sistema de Informações de Nascidos Vivos (SINASC), sendo coletadas informações relativas às características maternas, assistenciais e dos recém-nascidos cujos partos ocorreram neste município nos anos de 2001, 2005 e 2010.

O SINASC foi implantada no Brasil em 1990, sendo regulamentado o fluxo de coleta de dados e a periodicidade do envio das informações sobre nascidos vivos para o nível central no ano 2000. Embora seja apontada a melhoria da cobertura e da qualidade dos dados desde então ${ }^{15}$, recente estudo de revisão, que comparou a taxa de nascimentos pré-termo de estudos primários com a do SINASC, considerando o peso ao nascer, concluiu que este último não reflete a verdadeira dimensão dos nascimentos pré-termo no Brasil, por subestimar a prevalência até 2010, período em que o Sistema previa o registro da idade gestacional em categorias e não em semanas completas. Assim, foi adotada no presente estudo a aplicação do fator de correção sugerido no citado artigo. Para tal, meninos e meninas, separadamente, foram distribuídos em faixas de peso ao nascer, considerando intervalos de 100 gramas $^{16}$.

De acordo com a literatura e dados disponíveis no SINASC, foram investigadas associações entre nascimento pré-termo (variável dependente) e as seguintes variáveis explanatórias: anos de aprovação escolar da mãe (até sete, oito ou mais), idade materna em anos (até 19, 20 a 34, 35 ou mais), número de consultas pré-natais (até seis, sete ou mais), tipo de parto (vaginal, cesárea), local do parto (baixo risco público ou privado, alto 
risco), gemelaridade (sim, não), malformação ( sim, não). A variável cor não foi utilizada, pois no ano de 2001 esse dado era ignorado na maior parte dos casos, sendo a situação progressivamente melhor nos anos posteriores, aspecto que reduziria a validade das comparações pretendidas.

Para identificar os fatores de risco de nascimento pré-termo, realizou-se análise de regressão logística univariada e múltipla, estimando-se as razões de odds (e respectivos intervalos de confiança de $95 \%$ ) brutas e ajustadas para potenciais fatores de confusão. A análise múltipla foi guiada por modelo teórico hierarquizado construído para a presente pesquisa com base em estudo desenvolvido no sul do Brasil ${ }^{17}$, sendo constituído por três blocos de variáveis: o bloco um (fatores mais distais) continha variáveis sociodemográficas (anos de aprovação escolar e idade materna); do bloco dois constavam variáveis assistenciais (número de consultas pré-natais, tipo e local de parto) e no bloco três estavam as variáveis do recém-nascido (gestação gemelar e ocorrência de malformação).

Para entrar na análise multivariada como potencial fator de confusão das variáveis do mesmo bloco ou dos inferiores, adotou-se $\mathrm{p}<0,20$, exceto no caso da escolaridade, mantida no modelo, apesar de não ter alcançado este valor, para expressar nível socioeconômico, reconhecido como o determinante mais distal do desfecho em estudo. Para ser identificado como fator de risco, considerou-se como valor crítico $\mathrm{p}<0,05$, avaliado pelo teste Wald, após ajuste para os fatores do mesmo bloco e precedentes.

A tendência temporal dos fatores de risco identificados foi avaliada mediante regressão linear, sendo "ano de nascimento" a variável explanatória e o fator de risco a variável dependente. Admitiu-se tendência crescente ou decrescente quando o p-valor do teste t do parâmetro foi $<0,05$.

O presente estudo foi avaliado e aprovado por Comitê de Ética em Pesquisa local e respeitou as recomendações para pesquisas envolvendo seres humanos.

\section{Resultados}

As características sociodemográficas, assistenciais e referentes aos recém-nascidos nos anos estudados são apresentadas na Tabela 1.

Tabela 1. Características sociodemográficas, assistenciais e relativas ao recém-nascido, segundo ano de nascimento. Botucatu, 2001-2010.

\begin{tabular}{|c|c|c|c|c|c|c|}
\hline \multirow{2}{*}{ Variáveis } & \multicolumn{2}{|c|}{$2001(n=1677)$} & \multicolumn{2}{|c|}{$2005(n=1671)$} & \multicolumn{2}{|c|}{$2010(n=1644)$} \\
\hline & $\mathbf{N}$ & $\%$ & $\mathbf{N}$ & $\%$ & $\mathbf{N}$ & $\%$ \\
\hline \multicolumn{7}{|l|}{ Aprovação escolar (anos) } \\
\hline 8 ou mais & 892 & 56,3 & 1151 & 69,0 & 1264 & 76,9 \\
\hline Até 7 & 692 & 43,7 & 518 & 31,0 & 379 & 23,1 \\
\hline \multicolumn{7}{|l|}{ Idade (anos) } \\
\hline 20 a 34 & 1257 & 75,5 & 1283 & 76,8 & 1237 & 75,3 \\
\hline 35 ou mais & 154 & 9,2 & 173 & 10,3 & 209 & 12,7 \\
\hline Até 19 & 254 & 15,3 & 215 & 12,9 & 198 & 12,0 \\
\hline \multicolumn{7}{|l|}{ Consultas pré-natais } \\
\hline 7 ou mais & 1177 & 80,0 & 1324 & 79,2 & 1400 & 85,2 \\
\hline Até 6 & 294 & 20,0 & 347 & 20,8 & 243 & 14,8 \\
\hline \multicolumn{7}{|l|}{ Tipo de parto } \\
\hline Vaginal & 937 & 56,2 & 892 & 53,4 & 766 & 46,6 \\
\hline Cesárea & 729 & 43,8 & 779 & 46,6 & 878 & 53,4 \\
\hline \multicolumn{7}{|l|}{ Local de Nascimento } \\
\hline Baixo risco público/privado & 1078 & 64,3 & 1162 & 69,8 & 1103 & 67,9 \\
\hline Alto risco & 598 & 35,7 & 503 & 30,2 & 522 & 32,1 \\
\hline \multicolumn{7}{|l|}{ Gemelar } \\
\hline Não & 1640 & 98,0 & 1633 & 97,8 & 1612 & 98,1 \\
\hline Sim & 33 & 2,0 & 37 & 2,2 & 32 & 1,9 \\
\hline \multicolumn{7}{|l|}{ Malformação } \\
\hline Não & 1630 & 98,9 & 1549 & 98,7 & 1625 & 98,8 \\
\hline $\operatorname{Sim}$ & 18 & 1,1 & 22 & 1,3 & 19 & 1,2 \\
\hline
\end{tabular}


Independentemente do ano de nascimento, a maior parte das mães estava na faixa etária de 20 a 34 anos e tinha pelo menos oito anos de aprovação escolar. Houve redução da proporção de mães com baixa escolaridade, de $43,7 \%$ para $23,1 \%$; em geral, três em cada quatro mulheres tinham realizado sete ou mais consultas de prénatal; a proporção de cesárea foi crescente: $43,8 \%$ no início da década e 53,4\% no final. Houve redução da frequência de nascimentos no hospital de alto risco de 2001 para 2005, seguida de discreto aumento. A ocorrência de gravidez gemelar variou de $1,9 \%$ a $2,2 \%$ e de malformação entre $1,1 \%$ e $1,3 \%$ (Tabela 1 ).

Considerando as taxas de nascimento prétermo inicialmente obtidas (8,6\%, 9,6\% e 10,5\%) e as corrigidas $(12,5 \%, 12,0 \%$ e $13,2 \%)$, observou-se que, com a aplicação do fator de correção, as taxas aumentaram $20,5 \% \mathrm{em} 2001,20,0 \% \mathrm{em}$ 2005 e 31,2\% em 2010 (dados não apresentados em Tabela). Em termos de tendência, com base nos valores corrigidos, houve discreta queda de
2001 para 2005 e aumento de um ponto percentual de 2005 para 2010.

A Tabela 2 apresenta resultados das análises univariadas relativas à investigação de associação entre nascimento pré-termo e as variáveis independentes consideradas, segundo ano de estudo.

Associaram-se significativamente ao nascimento pré-termo a idade materna de 35 anos ou mais (2001 e 2010) e a cesárea (2005 e 2010); ter menos de sete consultas pré-natais, nascer em hospital de alto risco e gestação gemelar (2001, 2005 e 2010) e malformação (2001 e 2005) (Tabela 2).

A Tabela 3 evidencia que, após ajuste para os fatores do mesmo bloco e dos precedentes, foram confirmados como associados ao risco de nascimento pré-termo: a idade materna igual ou superior a 35 anos, embora sem alcançar significância estatística em $2005(\mathrm{p}=0,073)$ e 2010 $(\mathrm{p}=0,051)$, menos de sete consultas pré-natais, parto cesárea, nascer em hospital de alto risco e gemeralidade.

Tabela 2. Associação entre nascimento pré-termo e variáveis sociodemográficas (Bloco 1), assistenciais (Bloco 2) e do recém-nascido (Bloco 3), por ano de nascimento. Botucatu, 2001-2010.

\begin{tabular}{|c|c|c|c|c|c|c|}
\hline \multirow{2}{*}{ Variáveis } & \multicolumn{2}{|l|}{2001} & \multicolumn{2}{|c|}{$2005(n=1671)$} & \multicolumn{2}{|c|}{$2010(\mathrm{n}=1644)$} \\
\hline & OR (IC95\%) & $\mathbf{p}$ & OR (IC95\%) & $\mathbf{p}$ & OR (IC95\%) & $\mathbf{p}$ \\
\hline \multicolumn{7}{|l|}{ Bloco I } \\
\hline \multicolumn{7}{|c|}{ Aprovação escolar (anos) } \\
\hline 8 ou mais & 1 & & 1 & & 1 & \\
\hline Até 7 & $1,16(0,81-1,68)$ & 0,406 & $0,85(0,59-1,23)$ & 0,403 & $1,00(0,69-1,45)$ & 0,986 \\
\hline \multicolumn{7}{|l|}{ Idade (anos) } \\
\hline 20 a 34 & 1 & & 1 & & 1 & \\
\hline 35 ou mais & $2,34(1,44-3,80)$ & 0,001 & $1,51(0,93-2,44)$ & 0,089 & $1,54(0,99-2,38)$ & 0,052 \\
\hline Até 19 & $1,49(0,95-2,35)$ & 0,079 & $1,01(0,61-1,66)$ & 0,961 & $1,51(0,96-2,36)$ & 0,072 \\
\hline \multicolumn{7}{|l|}{ Bloco II } \\
\hline \multicolumn{7}{|c|}{ Consultas pré-natais } \\
\hline 7 ou mais & 1 & & 1 & & 1 & \\
\hline Até 6 & $3,06(2,01-4,63)$ & $<0,001$ & $6,56(4,67-9,21)$ & $<\mathbf{0 , 0 0 1}$ & $5,41(3,84-7,62)$ & $<0,001$ \\
\hline \multicolumn{7}{|l|}{ Tipo de parto } \\
\hline Vaginal & 1 & & 1 & & 1 & \\
\hline Cesárea & $1,20(0,85-1,69)$ & 0,286 & $1,89(1,35-2,64)$ & $<0,001$ & $2,00(1,43-2,80)$ & $<0,001$ \\
\hline \multicolumn{7}{|l|}{ Hospital } \\
\hline Baixo risco ${ }^{*}$ & 1 & & 1 & & 1 & \\
\hline Alto risco & $3,61(2,77-4,70)$ & $<0,001$ & $2,35(1,91-2,87)$ & $<0,001$ & $2,22(1,81-2,74)$ & $<0,001$ \\
\hline \multicolumn{7}{|l|}{ Bloco III } \\
\hline \multicolumn{7}{|l|}{ Gemelar } \\
\hline Não & 1 & & 1 & & 1 & \\
\hline Sim & $15,38(7,47-31,66)$ & $<\mathbf{0 , 0 0 1}$ & $22,93(11,27-46,66)$ & $<0,001$ & $12,07(5,89-24,75)$ & $<0,001$ \\
\hline \multicolumn{7}{|l|}{ Malformação } \\
\hline Não & 1 & & 1 & & 1 & \\
\hline Sim & $4,34(1,52-12,37)$ & 0,006 & $2,81(1,02-7,73)$ & 0,045 & $2,29(0,75-6,99)$ & 0,144 \\
\hline
\end{tabular}

"Público e privado. 
Ser adolescente não se associou ao nascimento pré-termo, mas no último ano considerado há tendência de aumento no risco $(\mathrm{p}=0,069)$. A magnitude dos efeitos dos cinco fatores de risco de nascimento pré-termo identificados variou de 1,54 (idade igual ou superior a 35 anos em 2010) a 16,44 (gemelaridade em 2005).
A Tabela 4 apresenta os resultados das análises de regressão linear que avaliaram a tendência dos determinantes de nascimento pré-termo identificados. Aumentaram na década a idade igual ou superior a 35 anos e o parto cesárea, enquanto que fazer até seis consultas pré-natais diminuiu significativamente no mesmo período.

Tabela 3. Associação entre nascimento pré-termo e variáveis sociodemográficas (Bloco 1), assistenciais (Bloco 2) e do recém-nascido (Bloco 3), por ano de nascimento, considerando-se valores de odds ratio ajustado. Botucatu, 2001-2010.

\begin{tabular}{|c|c|c|c|c|c|c|}
\hline \multirow{2}{*}{ Variáveis } & \multicolumn{2}{|l|}{2001} & \multicolumn{2}{|c|}{$2005(n=1671)$} & \multicolumn{2}{|c|}{$2010(n=1644)$} \\
\hline & ORA $^{*}($ IC95\%) & $\mathbf{p}$ & ORA $^{*}($ IC95\%) & $\mathbf{p}$ & ORA $^{*}($ IC95\%) & $\mathbf{p}$ \\
\hline \multicolumn{7}{|c|}{ Bloco I } \\
\hline \multicolumn{7}{|c|}{ Aprovação escolar (anos) } \\
\hline 8 ou mais & 1 & & 1 & & 1 & \\
\hline Até 7 & $1,14(0,79-1,65)$ & 0,475 & $0,85(0,59-1,23)$ & 0,374 & $0,95(0,65-1,39)$ & 0,798 \\
\hline \multicolumn{7}{|l|}{ Idade (anos) } \\
\hline 20 a 34 & 1 & & 1 & & 1 & \\
\hline 35 ou mais & $2,02(1,18-3,44)$ & 0,010 & $1,51(0,93-2,44)$ & 0,073 & $1,54(0,99-2,38)$ & 0,051 \\
\hline Até 19 & $1,25(0,76-2,05)$ & 0,365 & $1,01(0,61-1,66)$ & 0,912 & $1,52(0,96-2,40)$ & 0,069 \\
\hline \multicolumn{7}{|l|}{ Bloco II } \\
\hline \multicolumn{7}{|c|}{ Consultas pré-natais } \\
\hline 7 ou mais & 1 & & 1 & & 1 & \\
\hline Até 6 & $2,85(1,76-4,62)$ & $<0,001$ & $6,56(4,67-9,21)$ & $<0,001$ & $4,82(3,30-7,05)$ & $<0,001$ \\
\hline \multicolumn{7}{|l|}{ Tipo de parto } \\
\hline Vaginal & 1 & & 1 & & 1 & \\
\hline Cesárea & $1,69(1,07-2,69)$ & 0,024 & $1,89(1,35-2,64)$ & $<0,001$ & $2,41(1,66-3,49)$ & $<0,001$ \\
\hline \multicolumn{7}{|l|}{ Hospital } \\
\hline Baixo risco $^{* *}$ & 1 & & 1 & & 1 & \\
\hline Alto risco & $3,25(2,44-4,32)$ & $<0,001$ & $2,35(1,91-2,87)$ & $<0,001$ & $1,81(1,45-2,26)$ & $<0,001$ \\
\hline \multicolumn{7}{|l|}{ Bloco III } \\
\hline \multicolumn{7}{|l|}{ Gemelar } \\
\hline Não & 1 & & 1 & & 1 & \\
\hline Sim & $12,95(4,81-34,90)$ & $<0,001$ & $22,93(11,27-46,66)$ & $<0,001$ & $8,14(3,69-17,92)$ & $<0,001$ \\
\hline \multicolumn{7}{|l|}{ Malformação } \\
\hline Não & 1 & & 1 & & 1 & \\
\hline Sim & $2,54(0,51-12,62)$ & 0,253 & $2,81(1,02-7,73)$ & 0,328 & $1,41(0,41-4,83)$ & 0,578 \\
\hline
\end{tabular}

"Odds ratio ajustado. ${ }^{* *}$ Público e privado.

Tabela 4. Tendência dos determinantes do nascimento pré-termo identificados. Botucatu, 2001-2010.

\begin{tabular}{lccrr}
\hline \multicolumn{1}{c}{ Variável } & $\mathbf{2 0 0 1}(\%)$ & $\mathbf{2 0 0 5}(\%)$ & $\mathbf{2 0 1 0}(\mathbf{\%})$ & $\mathbf{p}^{*}$ \\
\hline Idade materna igual ou superior a 35 anos & 9,2 & 10,4 & 12,7 & 0,001 \\
Até seis consultas pré-natais & 20,0 & 20,8 & 14,8 & $<0,001$ \\
Parto cesárea & 43,8 & 46,6 & 53,4 & $<0,001$ \\
Nascer em hospital de alto risco & 35,7 & 30,2 & 32,1 & 0,233 \\
Gravidez gemelar & 2,0 & 2,2 & 1,9 & 0,929
\end{tabular}

"Teste t do parâmetro $\beta$. 
Gravidez gemelar e nascer em hospital de alto risco permaneceram estáveis.

\section{Discussão}

O presente estudo estimou a taxa de nascimento pré-termo no início, meio e final da primeira década do presente século, considerando os nascimentos de mulheres residentes e ocorridos no município de Botucatu/SP. As taxas variaram entre 12 e 13,2\%, com crescimento discreto (de 1,2 pontos percentuais) no período. Também, identificou cinco fatores associados ao maior risco de nascimento pré-termo, dos quais três (parto cesárea, nascimento em hospital de referência para gravidez de alto risco e menos de sete consultas de pré-natais) são passíveis de intervenções no âmbito dos serviços de saúde e estão ligados principalmente à qualidade da atenção obstétrica. Os outros dois fatores associados: idade materna igual ou superior a 35 anos e gemelaridade, são menos sensíveis a modificações por ações do setor saúde, por sua natureza sociocultural e biológica, respectivamente. O conhecimento produzido é importante para a definição de prioridades na alocação de recursos e para a seleção de intervenções viáveis dirigidas à redução do nascimento pré-termo, apontado como a principal causa de morte na infância em recente revisão sistemática ${ }^{12}$.

A validade dos resultados acima sintetizados é influenciada principalmente pela qualidade da informação sobre idade gestacional ao nascimento, que constitui um dos maiores desafios metodológicos em estudos epidemiológicos sobre nascimento pré-termo, visto que na comparação com estudos de base populacional o número de pré-termos estimados com base no SINASC tem sido menor ${ }^{13,16}$. Assim, a opção por utilizar metodologia para correção das estimativas ${ }^{16}$, no presente estudo, parece acertada. É interessante notar que o percentual de subestimação variou entre $20,0 \%$ e $31,2 \%$, conforme o ano, valores abaixo da taxa global de subestimação apontada para o Brasil $(38,0 \%)$. Este fato indica que simplesmente aplicar o percentual de subestimação observado em âmbito nacional às taxas de nascimento pré-termo calculadas com base no SINASC para localidades específicas não é adequado e que as estimativas corrigidas aqui apresentadas podem ser aceitas como as mais adequadas possíveis, levando-se em conta a fonte dos dados. A uniformidade metodológica para registro dos dados ao longo do período analisado e para aplicação do fator de correção asseguram a possibilidade de análise de tendência, tanto da taxa de nascimento pré-termo quanto do comportamento dos fatores de risco.

A correção das taxas não interfere nas análises para identificação de fatores associados a nascimentos pré-termos, uma vez que não se pode modificar a situação individual dos recém-nascidos com relação à prematuridade nos bancos de dados. Deve-se reconhecer a presença de falsos negativos, o que joga contra a identificação de fatores de risco e pode reduzir a magnitude dos riscos detectados. Assim, é possível que algumas das associações que não alcançaram significância estatística a alcançassem com a melhor definição da condição de pré-termo/termo, ou seja, com a redução dos falsos negativos.

Ainda com relação à validade interna dos resultados, caberia também avaliar a qualidade das informações referentes às demais variáveis utilizadas, o que não foi possível viabilizar. A favor da qualidade dos dados, destaca-se que para a grande maioria das variáveis utilizadas neste estudo são baixas as frequências de informações ignoradas, especialmente por que a quase totalidade dos nascimentos ocorreu em ambiente hospitalar, situação favorável à alimentação do Sistema de Informações, como já apontado por outros pesquisadores $^{15}$.

A taxa de nascimento pré-termo corrigida em crianças nascidas de mães residentes em Botucatu, em 2010 , foi de $13,2 \%$, um pouco acima da nacional - estimada em $11,7 \%$ - e menor que a reportada $(14,8 \%)$ para a cidade de Pelotas, no sul do país, nesse mesmo ano. Destaca-se que essas comparações são possíveis por que todas foram produzidas com dados do SINASC e com aplicação de fator de correção ${ }^{16}$. Os altos valores obtidos e a discreta tendência de elevação na década evidenciam que a prematuridade é um problema de saúde pública grave a demandar intervenções preventivas.

Dentre os fatores de risco identificados modificáveis por intervenções conduzidas pelos serviços de atenção primária à saúde, destaca-se a cesárea. As taxas de parto cirúrgico, que já eram elevadas no começo da década $(43,8 \%)$, cresceram significativamente no período, alcançando $53,4 \%$. Os demais fatores, como nascimentos em hospital de alto risco e número insuficiente de consultas pré-natais, tiveram evolução mais favorável no período. Investimentos na melhoria da qualidade da atenção obstétrica realizados no município, como hierarquização da atenção prénatal e ao parto, qualificação dos enfermeiros das 
unidades de saúde da família em atenção pré-natal e ampliação do número de equipes, podem ter contribuído para tal.

A associação entre cesárea e nascimento prétermo tem sido evidenciada mundialmente $\mathrm{e}^{11} \mathrm{e}$ revisão de estudos de base populacional indicou o aumento das cesáreas como um dos responsáveis pelo aumento dos nascimentos prematuros no Brasil ${ }^{18}$. Dados nacionais indicam que essas operações representam $87,5 \%$ dos partos do setor privado ${ }^{19}$, de modo que a hipermedicalização do parto, representada inclusive pelo uso abusivo da cesárea ${ }^{20}$, desponta como um dos graves problemas de saúde brasileiro. No contexto local, é essa também a realidade.

Visando reduzir as taxas de cesárea no Brasil, especialmente no setor privado, foi recentemente divulgada e deverá ser implementada até julho de 2015, a Resolução Normativa 368, de 6 de janeiro de 2015 que, entre outros aspectos, dispõe sobre o direito de acesso das beneficiárias à informação sobre os percentuais de cesáreas e partos normais, por operadora, por estabelecimento de saúde e por médico ${ }^{21}$, esperando-se subsidiar a decisão das mulheres quanto a escolha do profissional que a acompanhará no período da gravidez e parto. Estudo de abrangência nacional indicou que apenas 36\% das multíparas e 14,6\% das primíparas atendidas no setor privado, que manifestaram desejo de ter o parto por via vaginal no início da gestação, evoluíram para esse tipo de parto, sendo que a decisão pela cesárea foi tomada, em geral, no final da gravidez e independentemente de complicações que justificassem esse tipo de parto $^{19}$.

Os outros fatores de risco de nascimento pré-termo identificados pelo presente estudo são menos vulneráveis a modificação por ações dos serviços de saúde. O aumento da ocorrência de gravidez em mulheres com idade igual ou superior a 35 anos é um fenômeno socioeconômico e cultural ligado à melhoria da escolaridade e maior participação da mulher no trabalho formal. No Estado de São Paulo, dados das estatísticas do Registro Civil da Fundação Sistema Estadual de Naálise de Dados (SEADE) mostram o aumento na proporção de mães mais velhas (3039 anos):em 2011, 32,1\% das parturientes estavam nesta faixa etária, enquanto que no ano 2000 a taxa foi de $24,1 \%{ }^{22}$. Entretanto, tais mulheres estão mais expostas a complicações como diabetes gestacional e doença hipertensiva da gravidez, complicações que tendem a aumentar a taxa de nascimentos pré-termo ${ }^{7}$. Preparar-se para essa realidade é um desafio adicional aos serviços de saúde materno-infantil no município.

Outro caminho pelo qual a idade materna tardia pode influir na taxa de nascimento prétermo é a maior possibilidade de indicação de parto cesárea, quer em decorrência de eventuais complicações clínicas, quer em decorrência da própria idade, ou mesmo de forma eletiva ${ }^{7}$.

No sentido oposto, diminuiu significativamente no período estudado a gravidez na adolescência, fenômeno favorável e já observado para o Estado de São Paulo: em 1998, 20,2\% dos nascimentos foram de mães adolescentes; em 2011 esse percentual foi de $14,7 \%{ }^{22}$, valor pouco acima do encontrado no município de Botucatu (12,0\% em 2010).

Destaca-se, ainda, que na década, a chance de nascimento pré-termo no serviço de referência para gestação e parto de alto risco caiu à metade. A explicação pode estar na rede de atenção obstétrica existente no município anteriormente citada. Com a adesão municipal à Rede Cegonha em 2013, a expectativa é de redução progressiva do efeito deste fator.

A associação entre nascimento pré-termo e menos de sete consultas pré-natais identificada nos três anos estudados pode ser decorrente da forma como se organiza a atenção pré-natal no país, visto que os protocolos incluem retorno mensal até 28 semanas, quinzenal até 36 e semanais apenas após 36 semanas. Assim, na medida em que o parto ocorre pré-termo, diminui a possibilidade de a mulher ter passado por sete ou mais consultas. Neste caso, o menor número de atendimentos seria consequência e não causa do parto pré-termo.

$\mathrm{O}$ quinto fator associado com maior risco de nascimento pré-termo, gemelaridade, exerce o efeito de maior magnitude, aumentando 13 , 16 e 8 vezes a chance de nascimento pré-termo, conforme o ano considerado, corroborando a literatura que aponta consistentemente tal condição como fator de risco independente deste desfecho. A sobredistensão uterina, como a observada em situações de gestação múltipla, é uma das mais importantes causas de parto prematuro, especialmente nos países com maior desenvolvimento econômico, onde outros possíveis fatores estão sob controle ${ }^{1}$. Ressalte-se que a frequência desses casos ainda é baixa no município e não se identificou tendência de crescimento. 


\section{Conclusões}

A taxa de nascimentos pré-termo no município de Botucatu foi alta nos três anos estudados e em 2010 alcançou $13,2 \%$, valor pouco superior ao estimado para o país nesse mesmo ano. A tendência foi de discreta elevação, com aumento de 1,2 pontos percentuais de 2005 para 2010.

Independente da escolaridade e idade materna, do nascimento em hospital de referência para gestação e parto de alto risco e do número de consultas de pré-natal, a cesárea aumentou significativamente o risco de nascimento pré-termo, foi o fator de risco identificado mais frequente e que apresentou tendência de aumento do começo para o final da década.
Foram também identificados como fatores associados a maior risco de parto pré-termo: idade materna igual ou superior a 35 anos, com tendência crescente na década; nascimento em hospital de referência, estável no período; menos de sete consultas de pré-natal, decrescente e gemeralidade, também estável.

Considerando-se a possibilidade de redução por ações no âmbito da atenção à saúde, a alta frequência e a tendência de aumento na década, os resultados apoiam priorizar ações dirigidas especificamente à redução de cesáreas como forma de redução das taxas de nascimento pré-termo na cidade do estudo.

\section{Colaboradores}

B Balbi participou da concepção, delineamento e interpretação dos dados e da aprovação da versão a ser publicada. MABL Carvalhaes e CMGL Parada participaram de todas as etapas da construção do artigo e da aprovação da versão a ser publicada. 


\section{Referências}

1. Simmons LE, Rubens CE, Darmstadt GL, Gravett MG Preveting preterm birth and neonatal mortality: exploring the epidemiology, causes and interventions. Semin Perinatol 2010; 34(6):408-415.

2. Yazji I, Sodhi CP, Lee EK, Good M, Egan CE, Afrazi A, Neal MD, Jia H, Lin J, Ma C, Branca MF, Prindle T, Richardson WM, Ozolek J, Billiar TR, Binion DG, Gladwin MT, Hackam DJ. Endothelial TLR4 activation impairs intestinal microcirculatory perfusion in necrotizing enterocolitis via eNOS-NO-nitrite signaling. Proc Natl Acad Sci USA 2013; 110(23):9451-9456.

3. Morales DM, Townsend RR, Malone JP, Ewersmann CA, Macy EM, Inder TE, Limbrick DD. Alterations in protein regulators of neurodevelopment in the cerebrospinal fluid of infants with posthemorrhagic hydrocephalus of prematurity. Mol Cell Proteomics 2012 11(6):M111.011973.

4. McEvoy C, Venigalla S, Schilling D, Clay N, Spitale P, Nguyen T. Respiratory function in healthy late preterm infants delivered at 33-36 weeks of gestation. J Pediatr 2013; 162(3):464-469.

5. McAnulty G, Duffy FH, Kosta S, Weisenfeld NI, Warfield SK, Butler SC, Alidoost M, Bernstein JH, Robertson R, Zurakowski D, Als H. School-age effects of the newborn individualized developmental care and assessment program for preterm infants with intrauterine growth restriction: preliminary findings. $B M C$ Pediatr 2013; 13:25.

6. Teng RJ, Wu TJ. Persistent pulmonary hypertension of the newborn. J Formos Med Assoc 2013; 112(4):177184.

7. Beck S, Wojdyla D, Say L, Betran AP, Merialdi M, Requejo JH, Rubens C, Menon R, Van Look PFA. The worldwide incidence of preterm birth: a systematic review of maternal mortality and morbidity. Bull World Health Organ 2010; 88(1):31-38.

8. Silveira MF, Victora CG, Barros AJD, Santos IS, Matijasevich A, Barros FC. Determinants of preterm birth: Pelotas, Rio Grande do Sul State, Brasil, 2004 birth cohort. Cad Saude Publica 2010; 26(1):185-194.

9. Sanchez SE, Puente GC, Atencio G, Qiu C, Yanez D, Gelaye B, Williams MA. Risk of spontaneous preterm birth in relation to maternal depressive, anxiety, and stress symptoms. J Reprod Med 2013; 58(1-2):25-33.

10. Barros FC, Victora CG, Matijasevich A, Santos IS, Horta BL, Silveira MF, Barros AJD. Preterm births, low weight, and intrauterine growth restriction in three birth cohorts in Southern Brazil: 1982, 1993 and 2004. Cad Saude Publica 2008; 24(Supl. 3):s390-s398.

11. March of Dimes, PMNCH, Save the Children, World Health Organization (WHO). Born Too Soon: The Global Action Report on Preterm Birth. In: Howson CP, Kinney MV, Lawn JE, editors. Geneva: WHO; 2012.

12. Liu L, Oza S, Hogan D, Perin J, Rudan I, Lawn JE, Cousensw S, Mathers C, Black RE. Global, regional and national causes of child mortality in 2000-13, with projections to inform post 2015 priorities: an update systematic analysis. Lancet 2015; 385:430-440.
13. Pereira APE, Leal MC, Gama SGN, Domingues RMSM, Schilithz AOC, Bastos MH. Determinação da idade gestacional com base em informações do estudo Nascer no Brasil. Cad. Saude Publica 2014; 30(Supl 1):S59-S70.

14. Fundação Sistema Estadual de Análise de Dados (SE$\mathrm{ADE}$ ). Informações sobre os municípios paulistas. São Paulo: SEADE; 2013.

15. Jorge MHM, Laurenti R, Gotlieb SLD. Análise da qualidade das estatísticas vitais brasileiras: a experiência de implantação do SIM e do SINASC. Cien Saude Colet 2007; 12(3):643-654.

16. Silveira MF, Matijasevich A, Horta BL, Bettiol H, Barbieri MA, Silva AA, Rondó PHC, Lunardelli AN, Peres MA, Gurgel RQ, Cunha AL, Calvano LM, Amin Jr J, Leal MC, Matos ACG, Maranhão AG, Cortez-Escalante JJ, Barros AJD, Barros FC, Victora CG. Prevalência de nascimentos pré-termo por peso ao nascer: revisão sistemática. Rev Saude Publica 2013; 47(5):992-1003.

17. Santos HG, Andrade SM, Silva AMR, Carvalho WO, Mesas AE. Fatores de risco para mortalidade infantil em município do sul do Brasil: comparação de duas coortes em análise hierarquizada. Cad Saude Publica 2012; 28(10):1915-26.

18. Silveira MF, Santos IS, Barros AJD, Matijasevich A, Barros FC, Victora CG. Aumento da prematuridade no Brasil: revisão de estudos de base populacional. Rev Saude Publica 2008; 42(5):957-964.

19. Domingues RMSM, Dias MAB, Nakamura-Pereira M, Torres JÁ, D’Orsi J, Pereira APE, Schilithz AOC, Leal MC. Processo de decisão pelo tipo de parto no Brasil: da preferência inicial das mulheres à via de parto final. Cad Saude Publica 2014; 30(Supl. 1):S101-S106.

20. Victora CG, Aquino EML, Leal MC, Monteiro CA, Barros FC, Szwarcwald CL. Saúde de mães e crianças no Brasil: progressos e desafios. Saúde no Brasil 2. Lancet 2011; p. 32-46.

21. Brasil. Agência Nacional de Saúde Suplementar. Resolução Normativa n ${ }^{\circ} 368$, de 6 de janeiro de 2015. Diário Oficial da União 2015; 7 jan.

22. Victora CG, Barreto ML, Leal MC, Monteiro CA, Schimidt MI, Paim J, Bastos FI, Almeida C, Bahia L, Travassos C, Reichenheim M, Barros FC, The Lancet Brazil Series Group. Condições de saúde e inovações nas políticas de saúde no Brasil: o caminho a percorrer. Lancet 2011; Supl. Saúde no Brasil 6:90-102.

Artigo apresentado em 06/10/2014

Aprovado em 16/04/2015

Versão final apresentada em 18/04/2015 